Mathematical Research Letters 5, 63-82 (1998)

\title{
THE LONGEST INCREASING SUBSEQUENCE IN A RANDOM PERMUTATION AND A UNITARY RANDOM MATRIX MODEL
}

\author{
KURT JOHANSSON
}

\begin{abstract}
If $L_{N}$ is the expected length of the longest increasing subsequence in a random permutation, then $L_{N} \sim 2 \sqrt{N}$ as $N \rightarrow \infty$. We give a new proof of this result using a connection with a certain unitary random matrix model. The asymptotic formula is directly related to a third order phase transition in this model found by Gross and Witten.
\end{abstract}

\section{Preliminaries and main results}

Let $S_{N}$ be the group of all permutations of $\{1,2, \ldots, N\}$. We take the uniform distribution on $S_{N}$ as our probability distribution. If $\pi \in S_{N}$ we say that $\pi\left(i_{1}\right), \ldots, \pi\left(i_{k}\right)$ is an increasing subsequence in $\pi$ if $i_{1}<i_{2}<\cdots<i_{k}$ and $\pi\left(i_{1}\right)<\pi\left(i_{2}\right)<\cdots<\pi\left(i_{k}\right)$. We are interested in the random variable $\ell_{N}(\pi)$ the length of the longest increasing subsequence in $\pi$. Let $F_{N}(n)=P\left[\ell_{N}(\pi) \leq n\right]$ be its distribution function. For large $N$ the function $F_{N}(n)$ rises sharply from close to 0 to close to 1 when $n \sim 2 \sqrt{N}$. A consequence of this is that the expectation $L_{N}$ of $\ell_{N}(\pi)$ is asymptotically $2 \sqrt{N}$. The problem of the asymptotics of $L_{N}$ has a long history starting with Ulam, $[\mathrm{U}],[\mathrm{BB}]$ and Hammersley, $[\mathrm{Ha}]$, and there are now several proofs, see $[\mathrm{LS}],[\mathrm{VK}],[\mathrm{AD}],[\mathrm{DZ1}]$ and [Se1]. In the present paper we will show that the sharp transition of $F_{N}(n)$ around $n \sim 2 \sqrt{N}$ is associated with a certain third-order phase transition in a unitary random matrix model first studied by Gross and Witten, [GW], in connection with the large- $N$ limit of 2-d $U(N)$ lattice gauge theory. This connection is interesting since it connects the questions about the distribution of $\ell_{N}(\pi)$ with the asymptotic properties of large random matrices a field which has recently advanced considerably.

The connection is through the following formula of Odlyzko et al, [OPWW], and Rains, [Ra],

$$
F_{N}(n)=\frac{2^{2 N} N !}{(2 N) !} \mathcal{J}(2 N, n)
$$

Received September 3, 1997.

1991 Mathematics Subject Classification. Primary: 60B15, 60C05. Secondary: 05E15, $82 \mathrm{~B} 26$. 
where

$$
\begin{aligned}
\mathcal{J}(m, n) & =\frac{1}{(2 \pi)^{n} n !} \int_{[-\pi, \pi]^{n}}\left(\sum_{j=1}^{n} \cos \theta_{j}\right)^{m} \prod_{1 \leq j<k \leq n}\left|e^{i \theta_{j}}-e^{i \theta_{k}}\right|^{2} d^{n} \theta \\
& =\int_{U(n)}\left(\operatorname{Tr}\left(\frac{U+U^{*}}{2}\right)\right)^{m} d U .
\end{aligned}
$$

Here $d U$ denotes normalized Haar measure on the unitary group. The second equality in (1.2) follows from Weyls integration formula, [We]. The proof in [OPWW] starts from Gessel's generating function for $F_{N}(n)$, [Ge], see below. A nice derivation, using representation theory, has been given by Rains [Ra].

Clearly

$$
L_{N}=\sum_{n=1}^{N} n\left(F_{N}(n)-F_{N}(n-1)\right),
$$

and a summation by parts gives

$$
L_{N}=\sum_{n=0}^{N-1}\left(1-F_{N}(n)\right) .
$$

To simplify the asymptotic analysis we will now assume that $N$ is a Poisson random variable with mean $\lambda$, and consider the expectation (Poissonization)

$$
\phi_{n}(\lambda)=\sum_{N=0}^{\infty} \frac{e^{-\lambda} \lambda^{N}}{N !} F_{N}(n),
$$

where $F_{0}(n) \equiv 1$. We will show below that $F_{N}(n)$ is decreasing in $N$ for a fixed $n$, and hence from asymptotic information about $\phi_{n}(\lambda)$ we can extract asymptotic information about $F_{N}(n)$ (de-Poissonization). Since $\mathcal{J}(m, n)=0$ if $m$ is odd, we get

$$
\begin{aligned}
& \phi_{n}(\lambda)= \\
& \begin{aligned}
e^{-\lambda} \frac{1}{(2 \pi)^{n} n !} \int_{[-\pi, \pi]^{n}} \exp \left(2 \sqrt{\lambda} \sum_{j=1}^{n} \cos \theta_{j}\right) & \prod_{1 \leq j<k \leq n}\left|e^{i \theta_{j}}-e^{i \theta_{k}}\right|^{2} d^{n} \theta \\
& =e^{-\lambda} E_{n}\left(\exp \left(2 \sqrt{\lambda} \sum_{j=1}^{n} \cos \theta_{j}\right)\right),
\end{aligned}
\end{aligned}
$$

by combining $(1.1),(1.2)$ and $(1.4)$. Here $E_{n}(\cdot)$ denotes expectation with respect to the probability density

$$
\frac{1}{(2 \pi)^{n} n !} \prod_{1 \leq j<k \leq n}\left|e^{i \theta_{j}}-e^{i \theta_{k}}\right|^{2}
$$


on $[-\pi, \pi]^{n}$. This is the probability density for the eigenvalues of a unitary random matrix taken randomly with respect to normalized Haar measure on $U(n)$, see [Me]. It is the representation (1.5) that allows us to use random matrix theory. The expectation in the last expression in (1.5) can also be given another interpretation. It is equal to the $n \times n$ Toeplitz determinant, $D_{n}(f)$, with generating function $f(\theta)=\exp (2 \sqrt{\lambda} \cos \theta)$. Thus

$$
\phi_{n}(\lambda)=D_{n}\left(e^{2 \sqrt{\lambda} \cos \theta}\right) .
$$

Actually in going to formula (1.6) we have gone back to Gessel's generating function, see sect. 7 in $[\mathrm{Ge}]$ and $[\mathrm{GWW}]$; the elements in the Toeplitz determinant are $l_{j-k}(2 \sqrt{\lambda})$, where $l_{j}$ is the $j$ th modified Bessel function. This formula is interesting since it opens the possibility of investigating the asymptotic properties of $F_{N}(n)$ through asymptotic properties of Toeplitz determinants. It follows from Szegö's strong asymptotic formula for Toeplitz determinants, [Sz2], that $\phi_{n}(\lambda) \rightarrow 1$ as $n \rightarrow \infty$ for $\lambda$ fixed. In the present case we are interested in the case when $n$ and $\sqrt{\lambda}$ are of the same order, so we are considering a different type of asymptotics. From a statistical mechanical point of view we can think of (1.5) as the partition function of a Coulomb gas of unit charges on the unit circle with logarithmic repulsion and an external potential $2 \sqrt{\lambda} \cos \theta$. Consider the free energy,

$$
f(\gamma)=\lim _{n \rightarrow \infty} \frac{1}{n^{2}} \log E_{n}\left(\exp \left(\gamma n \sum_{j} \cos \theta_{j}\right)\right)
$$

of this Coulomb gas. We will show below, lemma 2.1, that $f(\gamma)=\gamma^{2} / 4$ if $0 \leq \gamma \leq 1$ and $f(\gamma)=\gamma-3 / 4-(\log \gamma) / 2$ if $\gamma>1$. Hence $d^{3} f / d \gamma^{3}$ is discontinuous at $\gamma=1$, and we have a third order phase transition, [GW]. For $\gamma<1$ the asymptotic eigenvalue distribution is supported on the whole unit circle, whereas for $\gamma>1$ it is supported on a subset of the circle. If we compare with (1.5) we see that the phase transition occurs when $n \sim 2 \sqrt{\lambda}$, and the analysis below will show that this entails that $F_{N}(n)$ rises sharply from 0 to 1 when $n \sim 2 \sqrt{N}$, and thus, by (1.3), we expect $L_{N} / \sqrt{N} \rightarrow 2$ as $N \rightarrow \infty$. Of course, to make this rigorous we have to make precise estimates. The main results are

Theorem 1.1. If $F_{N}(n)$ is the distribution of the length of the longest increasing subsequence in a random permutation from $S_{N}$, then

$$
\lim _{N \rightarrow \infty} F_{N}(x \sqrt{N})= \begin{cases}0 & \text { if } 0 \leq x<2 \\ 1 & \text { if } x>2\end{cases}
$$

We will also give a new proof of

Theorem 1.2. The expected length $L_{N}$ of the longest increasing subsequence in a random permutation in $S_{N}$ satisfies

$$
\lim _{N \rightarrow \infty} \frac{L_{N}}{\sqrt{N}}=2 .
$$


As stated above there are many proofs of this result, see [LS], [VK], [AD] and [Se1], and the purpose of the present paper is to show that the result can also be obtained using analytic tools from the theory of random matrices. Since there has recently been a lot of interest in random martices and many new results, this opens a new perspective on the problem of understanding the distribution of $\ell_{N}(\pi)$. The formulas (1.5) and (1.6) make it possible to use tools for asymptotic analysis that have been used in connection with random matrix problems. For example to get the variance of $\ell_{N}$ we need precise information about the behaviour of $\phi_{n}(\lambda)$ near the transition point. This is related to the so called double-scaling limit for the unitary random matrix model, see [PS]. A heuristic analysis using the free energy, lemma 2.1, shows that $\phi_{n}(\lambda$ should, as a function of $n$, rise from 0 to 1 in a region of size $\sim N^{1 / 6}$, which leads to $\operatorname{var}\left(\ell_{N}\right) \sim N^{1 / 3}$. That $\operatorname{var}\left(\ell_{N}\right)$ grows like $N^{1 / 3}$ was first conjectured by Odlyzko and Rains about 5 years ago on the basis of Monte Carlo simulations, [Od], see also [Ki] for a discussion of this conjecture. These Monte Carlo simulations also give more detailed information about the mean and the variance, see $[\mathrm{OR}]$. Work to prove this rigorously is in progress, [BDJ].

The theorems will be proved in the next section, but we postpone the proofs of several lemmas to sections 3 and 4 . The ideas used in the asymptotic analysis are closely related to those in [Jo], but a more refined analysis is needed in the present case.

\section{Proof of the main theorems}

Put

$$
G_{n}(\gamma)=E_{n}\left(\exp \left(\gamma n \sum_{j} \cos \theta_{j}\right)\right)
$$

for $\gamma \geq 0$, so that

$$
\phi_{n}(\lambda)=e^{-\lambda} G_{n}\left(\frac{2 \sqrt{\lambda}}{n}\right)
$$

We have

Lemma 2.1. [GW] If $f_{n}(\gamma)=n^{-2} \log G_{n}(\gamma)$, then

$$
\lim _{n \rightarrow \infty} f_{n}(\gamma)=f(\gamma)= \begin{cases}\gamma^{2} / 4 & \text { if } 0 \leq \gamma \leq 1 \\ \gamma-3 / 4-(\log \gamma) / 2 & \text { if } \gamma>1\end{cases}
$$

The proof will be given in section 3. Using lemma 2.1 we can prove

Lemma 2.2. Let $\epsilon>0$ be given. There are positive constants $C, \delta$, that only depend on $\epsilon$, such that if $n \leq(1+\epsilon)^{-1} 2 \sqrt{\lambda}$, then

$$
\phi_{n}(\lambda) \leq C e^{-\delta \lambda}
$$


Proof. Clearly $F_{N, n}$ is increasing in $n$, so the same is true for $\phi_{n}(\lambda)$ by (1.4). Hence, if we write $n(\lambda)=\left[(1+\epsilon)^{-1} 2 \sqrt{\lambda}\right]$, then

$$
\phi_{n}(\lambda) \leq \phi_{n(\lambda)}(\lambda)=e^{-\lambda} E_{n(\lambda)}\left(\exp \left(\frac{2 \sqrt{\lambda}}{n(\lambda)} n(\lambda) \sum_{j} \cos \theta_{j}\right)\right)
$$

Now,

$$
\left|\frac{2 \sqrt{\lambda}}{n(\lambda)}-(1+\epsilon)\right| \leq \frac{2}{n(\lambda)}
$$

if $\lambda$ is sufficiently large and $\epsilon \leq 1$. Consequently,

$$
\left|\frac{2 \sqrt{\lambda}}{n(\lambda)} n(\lambda) \sum_{j} \cos \theta_{j}-(1+\epsilon) n(\lambda) \sum_{j} \cos \theta_{j}\right| \leq 2 n(\lambda) \leq 4 \sqrt{\lambda}
$$

and the estimate (2.3) yields

$$
\phi_{n}(\lambda) \leq \exp \left(-\lambda+4 \sqrt{\lambda}+n(\lambda)^{2} f_{n(\lambda)}(1+\epsilon)\right) .
$$

Since $n(\lambda)^{2} / \lambda \rightarrow 4 /(1+\epsilon)^{2}$ as $\lambda \rightarrow \infty,(2.4)$ and lemma 2.1 give

$$
\limsup _{\lambda \rightarrow \infty} \phi_{n}(\lambda)^{1 / \lambda} \leq \exp \left(-1+\frac{4}{(1+\epsilon)^{2}} f(1+\epsilon)\right) .
$$

Expanding in a Taylor series we see that

$$
-1+\frac{4}{(1+\epsilon)^{2}} f(1+\epsilon)=-\frac{2}{3} \epsilon^{3}+\frac{11}{6} \epsilon^{4}-\ldots
$$

and thus, if $0<\epsilon<\epsilon_{0}, \epsilon_{0}$ sufficiently small, then

$$
\limsup _{\lambda \rightarrow \infty} \phi_{n}(\lambda)^{1 / \lambda} \leq \exp \left(-\epsilon^{3} / 3\right)
$$

It follows that $\phi_{n}(\lambda) \leq \exp \left(-\epsilon^{3} \lambda / 4\right)$ for all sufficiently large $\lambda$, and the lemma is proved.

Lemma 2.1 can be interpreted as a large deviation result for the Poissonized case,

$$
\lim _{\lambda \rightarrow \infty} \frac{1}{\lambda} \log \phi_{[x \sqrt{\lambda}]}(\lambda)=-1+2 x-\frac{3}{4} x^{2}+\frac{x^{2}}{2} \log \frac{x}{2},
$$

if $x<2$. A large deviation result for $\ell_{N}$ has recently been proved in [DZ2], see also $[\mathrm{Se} 2]$.

The proof of the next lemma is long and occupies a large part of the paper, so we postpone it to the next two sections. 
Lemma 2.3. Let $\epsilon>0$ be given. There is a constant $C$, which only depends on $\epsilon>0$, such that

$$
0 \leq 1-\phi_{n}(\lambda) \leq \frac{C}{n}
$$

if $n \geq \frac{2 \sqrt{\lambda}}{1-\epsilon}$.

The upper bound in (2.5) is not optimal. The methods of the present paper can be used to show that $1-\phi_{n}(\lambda) \leq C_{d} / n^{d}$ for any fixed $d$. A more precise large deviation result has recently been proved in [Se2], see also [DZ2]. Seppäläinen shows that

$$
\lim _{\lambda \rightarrow \infty} \frac{1}{\sqrt{\lambda}}\left(1-\phi_{[x \sqrt{\lambda}]}(\lambda)\right)=-2 x \cosh ^{-1}\left(\frac{x}{2}\right)+2 \sqrt{x^{2}-4},
$$

if $x \geq 2$.

In order to extract asymptotic information about $F_{N}(n)$ from $\phi_{n}(\lambda)$ we will use the fact that $F_{N}(n)$ is decreasing in $N$ for $n$ fixed:

Lemma 2.4. For all $n, N \geq 1$,

$$
F_{N+1}(n) \leq F_{N}(n)
$$

Using this lemma we can show the following "de-Poissonization"-lemma. Both the lemmas will be proved in section 4 .

Lemma 2.5. Write $\mu_{N}=N+4 \sqrt{N \log N}$ and $\nu_{N}=N-4 \sqrt{N \log N}$. Then there is a constant $C$ such that

$$
\phi_{n}\left(\mu_{N}\right)-\frac{C}{N^{2}} \leq F_{N}(n) \leq \phi_{n}\left(\nu_{N}\right)+\frac{C}{N^{2}}
$$

for all sufficiently large $N, 0 \leq n \leq N$

We can now give the

Proof of theorem 1.1. Let $x<2$. From (2.6) we get

$$
F_{N}([x \sqrt{N}]) \leq \phi_{[x \sqrt{N}]}\left(\nu_{N}\right)+\frac{C}{N^{2}}
$$

Since $[x \sqrt{N}] / \sqrt{\nu_{N}} \leq 1 /(1+\epsilon)$, we see from lemma 2.2 that, if we choose $\epsilon$ sufficiently small and $N$ sufficiently large,

$$
0 \leq F_{N}([x \sqrt{N}]) \leq C e^{-\delta \nu_{N}}+\frac{C}{N^{2}}
$$


This proves the first half of (1.7). In the case $x>2$ the first inequality in (2.6) gives

$$
\phi_{[x \sqrt{N}]}\left(\mu_{N}\right)-\frac{C}{N^{2}} \leq F_{N}([x \sqrt{N}]) \leq 1
$$

We have $[x \sqrt{N}] / \sqrt{\mu_{N}} \geq 1 /(1-\epsilon)$ if $\epsilon$ is small and $N$ large enough. Thus by lemma 2.3 and $(2.7)$,

$$
1-\frac{C}{[x \sqrt{N}]}-\frac{C}{N^{2}} \leq F_{N}(n) \leq 1
$$

which establishes the second half of theorem 1.1.

Next we prove theorem 1.2 by using the formula (1.3).

Proof of theorem 1.2. Fix $\epsilon>0$. Then, by (2.6) and lemma 2.2, $F_{N}(n) \leq C / N^{2}$ if $n \leq(1+\epsilon)^{-1} 2 \sqrt{N}$, and by (2.6) and lemma 2.3, $1-F_{N}(n) \leq C / N^{2}+C / n$, if $n \geq 2 \sqrt{N} /(1-\epsilon), N$ sufficiently large. Using (1.3) we thus have

$$
\left|L_{N}-2 \sqrt{N}\right| \leq C(\epsilon \sqrt{N}+\log N+1),
$$

and the theorem is proved since $\epsilon$ was arbitrary.

\section{Proofs of the asymptotic formulas}

Let $h_{n}(\theta)$ be a $2 \pi$-periodic function, which is $C^{1}$ and satisfies $\left|h_{n}^{\prime}(\theta)\right| \leq C$ for some constant $C, n \geq 1$. Let $u_{n}^{\xi, h_{n}}(t)$ be the 1-point function of the probability density

$$
\frac{1}{Z_{n}} \prod_{j \neq k}\left|e^{i \theta_{j}}-e^{i \theta_{k}}\right| \exp \left(\xi n \sum_{j} \cos \theta_{j}+\sum_{j} h_{n}\left(\theta_{j}\right)\right)
$$

on $[-\pi, \pi]^{n}$. Note that

$$
u_{n}^{0,0}(t)=\frac{1}{2 \pi}
$$

Now,

$$
\frac{d}{d \xi} \log G_{n}(\xi)=n^{2} \int_{-\pi}^{\pi} \cos t u_{n}^{\xi, 0}(t) d t
$$

so we need to understand the asymptotics of $u_{n}^{\xi, 0}$. Define for $|z|<1$,

$$
U_{n}(z)=\int_{-\pi}^{\pi} \frac{u_{n}(t)}{1-z e^{-i t}} d t
$$


(we suppress the upper indices of $u_{n}$ ), and

$$
H_{n}(z)=\int_{-\pi}^{\pi} \frac{-i h_{n}(t) u_{n}(t)}{1-z e^{-i t}} d t
$$

Let $k_{n}(t, s)=n u_{n}(t) u_{n}(s)-(n-1) u_{n}(t, s)$, where $u_{n}(t, s)$ is the two-point function of (3.1), and

$$
\begin{aligned}
& K_{n}(z)= \\
& \quad n \int_{-\pi}^{\pi} \int_{-\pi}^{\pi}\left(\frac{1}{\left(1-z e^{-i t}\right)\left(1-z e^{-i s}\right)}-\frac{1}{\left(1-z e^{-i t}\right)^{2}}\right) k_{n}(t, s) d t d s .
\end{aligned}
$$

We will show in section 4 that $U_{n}$ satisfies the equation

$$
n U_{n}(z)^{2}-n\left(\frac{\xi}{2}\left(z-\frac{1}{z}\right)+1\right) U_{n}(z)-\frac{1}{2} \xi n c_{n}-\frac{\xi n}{2 z}=\frac{1}{n} K_{n}(z)-H_{n}(z),
$$

where

$$
c_{n}=\int_{-\pi}^{\pi} e^{i t} u_{n}(t) d t
$$

Lemma 3.1. Let $\delta>0$. If $|z| \leq 1-\delta$ and $n \geq 1$, then

$$
\left|K_{n}(z)\right| \leq \frac{2 n}{\delta^{2}}
$$

Proof. Let $\left\{p_{n, k}\left(e^{i \theta}\right)\right\}_{k=0}^{\infty}$ be the sequence of orthonormal polynomials on the unit circle with respect to the weight $w_{n}(\theta)=\exp \left(\gamma_{n} n \cos \theta+h_{n}(\theta)\right)$. Then, $[\mathrm{Me}]$,

$$
k_{n}(t, s)=\frac{1}{n}\left|\sum_{k=0}^{n-1} p_{n, k}\left(e^{i t}\right) p_{n, k}\left(e^{i s}\right)\right|^{2} w_{n}(t) w_{n}(s),
$$

so $k_{n} \geq 0$. Also, $\int k_{n}=1$, so the result follows immediately by estimating the integral in (3.4).

The sequence $\left\{U_{n}\right\}$ is a normal family in $|z|<1$. If $U_{n_{j}}$ is a convergent subsequence, then the limit $U$ must satisfy the equation

$$
U(z)^{2}-\left(\frac{\xi}{2}\left(z-\frac{1}{z}\right)+1\right) U(z)-\frac{\xi}{2} \frac{1}{z}-\frac{\xi}{2} c=0,
$$

where

$$
U(z)=\int_{-\pi}^{\pi} \frac{d \mu(t)}{1-z e^{-i t}},
$$

for some probability measure $d \mu$, which is the weak-*-limit of $u_{n_{j}} d t$; $c=\int_{[-\pi, \pi]} \exp (i t) d \mu(t)$. This follows from (3.5) by dividing with $n$, letting $n_{j} \rightarrow \infty$, using $\left|H_{n}(z)\right| \leq C / \delta$ for $|z| \leq 1-\delta$ and lemma 3.1. 
Lemma 3.2. ([GW]) Suppose that $U(z)$ is given by (3.7) and satisfies the equation (3.6) with $\xi \geq 0$ and $c$ a constant.

(i) If $\xi \leq 1$, then $U(z)=1+\xi z / 2, c=\xi / 2$ and $d \mu(t)=(2 \pi)^{-1}(1+\xi \cos t) d t$.

(ii) If $\xi>1, d \mu(t)=\pi^{-1} \xi \cos (t / 2) \sqrt{1 / \xi-\sin ^{2}(t / 2)} \chi_{\left[-t_{c}, t_{c}\right]}(t) d t$, where $\sin ^{2}\left(t_{c} / 2\right)=1 / \xi, 0 \leq t_{c}<\pi$ and $c=1-1 /(2 \xi)$.

The proof will be given in section 4 . Since the limit $U$ is unique it follows that $U_{n} \rightarrow U$ as $n \rightarrow \infty$ and

$$
\int_{-\pi}^{\pi} \cos t u_{n}^{\xi, 0}(t) d t \rightarrow \psi(t)= \begin{cases}\xi / 2 & \text { if } 0 \leq \xi \leq 1, \\ 1-1 /(2 \xi) & \text { if } \xi>1 .\end{cases}
$$

(Note that $u_{n}^{\xi, 0}$ is an even function.)

We are now ready for the

Proof of lemma 2.1. It follows from (3.3) that $f_{n}^{\prime}(\xi)=\int_{[-\pi, \pi]} \cos t u_{n}^{\xi, 0}(t) d t$, and hence $\left|f_{n}^{\prime}(\xi)\right| \leq 1$. Since $f_{n}^{\prime} \rightarrow \psi$ as $n \rightarrow \infty$ in $[0, \gamma]$, the dominated convergence theorem gives

$$
f_{n}(\gamma)=\int_{0}^{\gamma} f_{n}^{\prime}(\xi) d \xi \rightarrow \int_{0}^{\gamma} \psi(\xi) d \xi=f(\gamma)
$$

and we are done.

Next, we give the

Proof of lemma 2.3. Write $u^{\xi}(t)=(2 \pi)^{-1}(1+\xi \cos t)$. Then, by (3.3),

$$
\frac{d}{d \xi} \log G_{n}(\xi)=n^{2} \int_{-\pi}^{\pi} \cos t\left(u_{n}^{\xi, 0}(t)-u^{\xi}(t)\right) d t+n^{2} \frac{\xi}{2} .
$$

Put

$$
A_{n}(\xi)=n^{3}\left|\int_{-\pi}^{\pi} \cos t\left(u_{n}^{\xi, 0}(t)-u^{\xi}(t)\right) d t\right| .
$$

It follows from (3.2) that $A_{n}(0)=0$. Let $\epsilon>0$ be given and pick $\gamma \in[0,1-\epsilon]$. Integration of (3.9) from 0 to $\gamma$ gives

$$
\left|\log G_{n}(\gamma)-\frac{n^{2} \gamma^{2}}{4}\right| \leq \frac{1}{n} \int_{0}^{\gamma} A_{n}(\xi) d \xi .
$$

From the definition it is clear that $A_{n}(\xi)$ is a continuous function on $[0,1-\epsilon]$ and hence

$$
\max _{0 \leq \xi \leq 1-\epsilon} A_{n}(\xi)=A_{n}\left(\gamma_{n}\right)
$$

for some $\gamma_{n} \in(0,1-\epsilon]$. We can assume that $\gamma_{n}>0$ since $A_{n}(0)=0$ and $A_{n}(\xi) \geq 0$. The inequality (3.10) gives

$$
\left|\log G_{n}(\gamma)-\frac{n^{2} \gamma^{2}}{4}\right| \leq \frac{1}{n} A_{n}\left(\gamma_{n}\right),
$$


if $\gamma \in[0,1-\epsilon]$. Let $\lim \sup _{n \rightarrow \infty} A_{n}\left(\gamma_{n}\right)=\lim _{k \rightarrow \infty} A_{n_{k}}\left(\gamma_{n_{k}}\right)$, where we can assume that $\gamma_{n_{k}} \rightarrow \gamma_{0} \in[0,1-\epsilon]$, after, perhaps, picking yet another subsequence. To simplify notation we will write $\gamma_{n}$ instead of $\gamma_{n_{k}}$. The key result is the next lemma which we will prove below.

Lemma 3.3. Let $\epsilon>0$ be given. If $0<\gamma_{n} \leq 1-\epsilon$ and $\gamma_{n} \rightarrow \gamma_{0}$ as $n \rightarrow \infty$, then there is a constant $C$, which only depends on $\epsilon$, such that $A_{n}\left(\gamma_{n}\right) \leq C$ for all $n \geq 1$.

If we accept lemma 3.3, the inequality (3.11) gives

$$
\left|\log G_{n}(\gamma)-\frac{n^{2} \gamma^{2}}{4}\right| \leq \frac{C}{n}
$$

If $n \geq 2 \sqrt{\lambda} /(1-\epsilon)$, we can take $\gamma=2 \sqrt{\lambda} / n$ and get

$$
\left|\log G_{n}(2 \sqrt{\lambda} / n)-\lambda\right| \leq C / n,
$$

which, using (2.1), proves lemma 2.3.

Proof of lemma 3.3. Below $h_{n}$ will be either identically zero, or a certain $2 \pi$ periodic $C^{1}$-function, (4.3), on $\mathbb{R}$ satisfying

$$
\left\|h_{n}^{\prime}\right\|_{\infty} \leq C, \quad n \geq 1
$$

for some constant $C$. Here and in what follows $C$ denotes a constant which only depends on $\epsilon$, but which may vary from place to place. Then

$$
\left|H_{n}(z)\right| \leq \frac{C}{\delta}, \quad|z| \leq 1-\delta .
$$

Observe that $U^{\gamma_{n}}$ satisfies

$$
U^{\gamma_{n}}(z)^{2}-\left(\frac{\gamma_{n}}{2}\left(z-\frac{1}{z}\right)+1\right) U^{\gamma_{n}}(z)-\frac{\gamma_{n}}{2} \frac{1}{z}-\frac{\gamma_{n}^{2}}{4}=0 .
$$

Put

$$
D_{n}(z)=D_{n}^{\gamma_{n}, h_{n}}(z)=n\left(U_{n}^{\gamma_{n}, h_{n}}(z)-U^{\gamma_{n}}(z)\right) .
$$

Taking the difference between the two equations (3.5) and (3.14) we see that $D_{n}$ satisfies

$$
\frac{1}{n^{2}} D_{n}^{2}-\left(\frac{\gamma_{n}}{2}\left(z+\frac{1}{z}\right)+1\right) \frac{D_{n}}{n}-x_{n}=\frac{1}{n^{2}} K_{n}-\frac{1}{n} H_{n}
$$

where $x_{n}=\gamma_{n}\left(c_{n}-\gamma_{n} / 2\right) / 2$. Let $z_{n}=-1 / \gamma_{n}+\sqrt{1 / \gamma_{n}^{2}-1}$, which is the root of $\gamma_{n}(z+1 / z) / 2+1=0$ that lies inside the unit circle. Since $0<\gamma_{n} \leq 1-\epsilon$ for all $n$ we can choose $\delta, 0<\delta<1 / 4$, so that $0<\left|z_{n}\right|<1-2 \delta$ for all $n \geq 1$. 
Equation (3.15) can be written

$$
\frac{1}{n} D_{n}=\frac{x_{n}+K_{n} / n^{2}-D_{n}^{2} / n^{2}-H_{n} / n}{\gamma_{n}(z+1 / z) / 2+1} .
$$

Let

$$
M_{n}=\sup _{|z|=1-\delta}\left|\frac{1}{n} D_{n}(z)\right| .
$$

From equation (3.16), lemma 3.1 and inequality (3.13) we find

$$
M_{n} \leq L\left(\left|x_{n}\right|+1 / n+M_{n}^{2}\right)
$$

where the constant $L$ only depends on $\epsilon$, and we can assume $L \geq 1$. We now claim that there is an $n_{0} \geq 16 L^{2}$ such that if $n \geq n_{0}$, then

(i) $\left|x_{n}\right| \leq 1 / 16 L^{2}$

(ii) $\left|M_{n}\right| \leq 1 / 16 L^{2}$.

To see this let

$$
\eta_{n}=\sup _{|z| \leq 1-\delta}\left|\frac{1}{n} D_{n}(z)\right|, \quad n \geq 1,
$$

which gives a bounded sequence. Assume that there is a subsequence $\eta_{n_{k}}$ such that $\eta_{n_{k}} \rightarrow \eta>0$ as $n_{k} \rightarrow \infty$. Since $\left\{U_{n_{k}}\right\}$ is a normal family in the open unit disk, we can extract a subsequence $\left\{U_{n_{k_{j}}}\right\}$ which converges uniformly in $|z| \leq 1-\delta$. It follows from equation (3.5) and lemma 3.2 that the limit must be $U^{\gamma_{0}}(z)=1+\gamma_{0} z / 2$, and since $\gamma_{n} \rightarrow \gamma_{0}$ it follows that $\eta_{n_{k_{j}}} \rightarrow 0$ as $j \rightarrow \infty$, and we have a contradiction. Hence $\eta_{n} \rightarrow 0$ as $n \rightarrow \infty$ and we can pick $n_{0}$ so that $\left|D_{n}^{\gamma_{n}, h_{n}}(z) / n\right| \leq 1 /\left(16 L^{2}\right)$ if $|z| \leq 1-\delta$ and $n \geq n_{0} ; n_{0}$ can be chosen so that this hold for both choices of $h_{n}$. Now,

$$
x_{n}=\frac{\gamma_{n}}{4 \pi i} \int_{|z|=1-\delta} \frac{D_{n}(z) / n}{z^{2}} d z
$$

and thus $\left|x_{n}\right| \leq 1 / 16 L^{2}$ if $n \geq n_{0}$. This proves (i) and (ii).

Using (i) it follows from (3.17) that

$$
M_{n} \leq 2 L\left(\left|x_{n}\right|+1 / n\right), \quad n \geq n_{0} .
$$

Since $\left|z_{n}\right|<1-\delta$ the maximum principle gives $\left|D_{n}\left(z_{n}\right)\right| / n \leq 2 L\left(\left|x_{n}\right|+1 / n\right)$, and hence taking $z=z_{n}$ in (3.15) we obtain

$$
\left|x_{n}\right| \leq 4 L^{2}\left(\left|x_{n}\right|+1 / n\right)^{2}+2 /\left(n \delta^{2}\right)+C / n,
$$

or

$$
\left|x_{n}\right|^{2}-\left(1 / 4 L^{2}-2 / n\right)\left|x_{n}\right|+C / n \geq 0
$$

Together with the estimate (ii) above this gives $\left|x_{n}\right| \leq C / n$ and thus

$$
\left|D_{n}^{\gamma_{n}, h_{n}}(z)\right| \leq C,
$$

if $|z| \leq 1-\delta$ and $n \geq n_{0}$.

The inequality (3.18), with $h_{n}$ given by (4.3) below, is the basis for the proof of the next lemma. We postpone the proof to section 4 . 
Lemma 3.4. There is a constant $C$ such that

$$
\left|K_{n}^{\gamma_{n}, 0}(z)\right| \leq C
$$

for all $|z| \leq 1-\delta$ and $n \geq n_{0}$.

Now, take $h_{n} \equiv 0$ so that $H_{n} \equiv 0$ in (3.15), and put $z=z_{n}$. The estimates (3.18) and (3.19) then give $\left|n x_{n}\right| \leq C / n, n \geq n_{0}$. Combining this estimate with equation (3.16), the estimates (3.18), (3.19) and the maximum principle, we get

$$
\left|D_{n}^{\gamma_{n}, 0}(z)\right| \leq C / n,
$$

for all $|z| \leq 1-\delta$ and $n \geq n_{0}$.

To get further we need a better estimate of $K_{n}^{\gamma_{n}, 0}$ than the one in lemma 3.4, and below we will prove

Lemma 3.5. There is a constant $C$ such that

$$
\left|K_{n}^{\gamma_{n}, 0}(z)\right| \leq C / n
$$

for all $|z| \leq 1-3 \delta / 2$ and $n \geq n_{0}$.

We can now repeat the same type of argument one more time. Equation (3.15) with $z=z_{n}$ together with the estimates (3.20) and (3.21) give $\left|n^{2} x_{n}\right| \leq C / n$. The same estimates and equation (3.15) then yield

$$
\left|n^{2} D_{n}^{\gamma_{n}, 0}(z)\right| \leq C, \quad n \geq n_{0},
$$

for $|z|=1-3 \delta / 2$.

Since $u_{n}^{\gamma_{n}, 0}(t)$ is even a straightforward computation shows that

$$
A_{n}\left(\gamma_{n}\right)=\frac{1}{2 \pi i} \int_{|z|=1-3 \delta / 2} \frac{n^{2} D_{n}(z)}{z^{2}} d z
$$

Combining this with (3.22), lemma 3.3 follows.

\section{The variational formulas and proofs of some lemmas}

Let $h$ and $g$ be two given $2 \pi$-periodic, $C^{1}$-functions. In the integral

$$
Z_{n}=\int_{[-\pi, \pi]^{n}}\left(\sum_{j} g\left(\theta_{j}\right)\right) e^{\sum_{j \neq k} \log \left|2 \sin \frac{\theta_{j}-\theta_{k}}{2}\right|+\xi n \sum_{j} \cos \theta_{j}+\sum_{j} h_{n}\left(\theta_{j}\right)} d^{n} \theta
$$

we make the change of variables $\theta_{j}=x_{j}+\epsilon \psi\left(x_{j}\right)$, where $\psi$ is a $2 \pi$-periodic, $C^{1}$-function with $\|\left.\epsilon \psi^{\prime}\right|_{\infty}<1$. The obvious equality $\left.\frac{d}{d \epsilon} \log Z_{n}\right|_{\epsilon=0}=0$ yields

$$
\begin{aligned}
E_{n}^{\gamma_{n}, h_{n}}\left[\left(\frac{1}{2} \sum_{j \neq k} \cot \left(\frac{x_{j}-x_{k}}{2}\right)\left(\psi\left(x_{j}\right)-\psi\left(x_{k}\right)\right)\right.\right. \\
-\xi n \sum_{j} \psi\left(x_{j}\right) \sin x_{j} \sum_{j} h_{n}^{\prime}\left(x_{j}\right) \psi\left(x_{j}\right) \\
\left.\left.\quad+\sum_{j} \psi^{\prime}\left(x_{j}\right)\right)\left(\sum_{\ell} g\left(x_{\ell}\right)\right)+\sum_{\ell} g^{\prime}\left(x_{\ell}\right) \psi\left(x_{\ell}\right)\right]=0 .
\end{aligned}
$$


In our first application of (4.1) we choose $g(\theta)=1 / n$, and obtain the identity

$$
\begin{aligned}
& \frac{1}{2} n(n-1) \int_{-\pi}^{\pi} \int_{-\pi}^{\pi} \cot \frac{t-s}{2}(\psi(t)-\psi(s)) u_{n}(t, s) d t d s \\
& -\xi^{2} n^{2} \int_{-\pi}^{\pi} \sin t \psi(t) u_{n}(t) d t+n \int_{-\pi}^{\pi} h_{n}^{\prime}(t) \psi(t) u_{n}(t) d t \\
& \quad+n \int_{-\pi}^{\pi} \psi^{\prime}(t) u_{n}(t) d t=0
\end{aligned}
$$

where $u_{n}(t, s)$ is the 2-point function of (3.1). Now, choose $\psi(t)=\left(1-z e^{-i t}\right)^{-1}$ in (4.2). This gives equation (3.5) after some algebraic manipulation.

Next, we will give the

Proof of lemma 3.3. Let $\Omega=\{|z| \leq 1-\delta\} \times\left\{\left|w_{1}\right| \leq 1\right\} \times\left\{\left|w_{2}\right| \leq 1\right\} \subseteq \mathbb{C}^{3}$ and $g_{n}\left(\theta ; z, w_{1}, w_{2}\right)=w_{1}\left(e^{i \theta}-\int_{-\pi}^{\pi} e^{i t} u^{\gamma_{n}}(t) d t\right)+w_{2}\left(\frac{1}{1-z e^{-i \theta}}-\int_{-\pi}^{\pi} \frac{u^{\gamma_{n}}(t)}{1-z e^{-i t}} d t\right)$.

On $\Omega$ we define

$$
F_{n}\left(z, w_{1}, w_{2}\right)=E_{n}^{\gamma_{n}, 0}\left(\exp \left(\sum_{j} g_{n}\left(\theta_{j} ; z, w_{1}, w_{2}\right)\right)\right)
$$

where $E_{n}^{\gamma_{n}, 0}(\cdot)$ denotes expectation wih respect to $(3.1)$ with $h_{n}=0$. Below we omit the superscripts. Then

$$
\left|F_{n}\left(z, w_{1}, w_{2}\right)\right| \leq E_{n}\left(\exp \left(\sum_{j} \operatorname{Re} g_{n}\left(\theta_{j} ; z, w_{1}, w_{2}\right)\right)\right) .
$$

The right hand side is a continuous function of $\left(z, w_{1}, w_{2}\right)$ in $\Omega$, a compact set, so it assumes its maximum at some point $\left(z^{(n)}, w_{1}^{(n)}, w_{2}^{(n)}\right)$. Write

$$
h_{n}(\theta)=\operatorname{Re} g_{n}\left(\theta ; z^{(n)}, w_{1}^{(n)}, w_{2}^{(n)}\right),
$$

and consider

$$
f_{n}(\theta)=\log E_{n}\left(\exp \left(\xi \sum_{j} h_{n}\left(\theta_{j}\right)\right)\right)
$$

for $0 \leq \xi \leq 1$. It is easy to see that $f_{n}^{\prime \prime}(\xi) \geq 0$, so $f_{n}^{\prime}(\xi)$ is increasing and consequently

$$
f_{n}(1)=\int_{0}^{1} f_{n}^{\prime}(\xi) d \xi \leq f_{n}^{\prime}(1)
$$

Now,

$$
\begin{aligned}
f_{n}^{\prime}(1) & =n \int_{-\pi}^{\pi} h_{n}(t) u_{n}^{\gamma_{n}, h_{n}}(t) d t \\
& =\operatorname{Re}\left(w_{1}^{(n)} \frac{1}{2 \pi i} \int_{|\zeta|=1-\delta} \frac{D_{n}^{\gamma_{n}, h_{n}}(\zeta)}{\zeta^{2}} d \zeta+w_{2}^{(n)} D_{n}^{\gamma_{n}, h_{n}}\left(z^{(n)}\right)\right),
\end{aligned}
$$


so using the inequality (3.18) we obtain

$$
\left|F_{n}\left(z, w_{1}, w_{2}\right)\right| \leq C
$$

for all $\left(z, w_{1}, w_{2}\right)$ in $\Omega$ and $n \geq n_{0}$. Put $G_{n}(w)=F_{n}(z, 0, w)$ for a fixed $z$. Cauchy's integral formula gives $\left|G_{n}^{\prime}(0)\right| \leq 4 C$ and $\left|G_{n}^{\prime \prime}(0)\right| \leq 8 C$, and since $K_{n}^{\gamma_{n}, 0}(z)=-\left(G_{n}^{\prime \prime}(0)-G_{n}^{\prime}(0)^{2}\right)$, we obtain $\left|K_{n}^{\gamma_{n}, 0}(z)\right| \leq 24 C^{2}$, and the lemma is proved.

We will now use equation (4.1) with other choices of of $g$ but with $h_{n}=0$. Write $r(z)=1+\gamma_{n}(z-1 / z) / 2$ and choose $\psi(t)=\left(1-z e^{-i t}\right)^{-1}$ in (4.1). This gives

$$
\begin{aligned}
E_{n} & {\left[\left(-\sum_{j, k} \frac{1}{\left(1-z e^{-i x_{j}}\right)\left(1-z e^{-i x_{k}}\right)}+n r(z) \sum_{j} \frac{1}{\left(1-z e^{-i x_{j}}\right)}\right.\right.} \\
& \left.\left.+\frac{\gamma n}{2} \sum_{j} e^{i x_{j}}+\frac{\gamma n^{2}}{2 z}\right)\left(\sum_{j} g\left(x_{j}\right)\right)-i \sum_{j} g^{\prime}\left(x_{\ell}\right) \frac{1}{\left(1-z e^{-i x_{j}}\right)}\right]=0 .
\end{aligned}
$$

Write $F=\sum_{j}\left(1-z e^{-i x_{j}}\right)^{-1}$ and $G=\sum_{j} g\left(x_{j}\right)$. Define

$$
\begin{aligned}
& L_{n}(z ; g)=E_{n}(F G)-E_{n}(F) E_{n}(G), \\
& K_{n}^{(2)}(z)=E_{n}\left(F^{2}\right)-E_{n}(F)^{2} \text { and } \\
& K_{n}^{(3)}(z, g)=E_{n}\left(F G^{2}\right)-E_{n}\left(F^{2}\right) E_{n}(G)-2 E_{n}(F) E_{n}(F G)+2 E_{n}(F)^{2} E_{n}(G) .
\end{aligned}
$$

Note that $K_{n}^{(2)}(z)=-K_{n}^{\gamma_{n}, 0}(z)$. Furthermore, let

$$
\mu_{n}(g)=\int_{-\pi}^{\pi} g(t) u_{n}^{\gamma_{n}, 0}(t) d t
$$

and

$$
\nu_{n}(g)=E_{n}\left(\left(\sum_{j} e^{i x_{j}}\right) G\right)
$$

Equation (4.1) can now be written

$$
\begin{aligned}
& -K_{n}^{(3)}(z, g)-n^{3}\left[\frac{1}{n^{2}} K_{n}^{(2)}(z)+U_{n}(z)^{2}-r(z) U_{n}(z)\right. \\
& \left.-\frac{\gamma_{n}}{2} c_{n}-\frac{\gamma_{n}}{2 z}\right] \mu_{n}(g)-\frac{\gamma_{n} n^{3}}{2} c_{n} \mu_{n}(g)-n\left(2 U_{n}(z)\right. \\
& -r(z)) L_{n}(z ; g)+\frac{\gamma_{n} n}{2} \nu_{n}(g)-i n \int_{-\pi}^{\pi} \frac{g^{\prime}(t) u_{n}(t)}{1-z e^{-i t}} d t=0 .
\end{aligned}
$$


Using equation (3.5) this can be simplified to

$$
\begin{aligned}
-K_{n}^{(3)}(z ; g)-n\left[\left(2 U_{n}(z)-r(z)\right)\right. & L_{n}(z ; g)-\frac{\gamma_{n} n^{3}}{2} \mu_{n}(g) c_{n} \\
& +\frac{\gamma_{n} n}{2} \nu_{n}(g)-i n \int_{-\pi}^{\pi} \frac{g^{\prime}(t) u_{n}(t)}{1-z e^{-i t}} d t=0 .
\end{aligned}
$$

If we choose $g(t)=\left(1-z e^{-i t}\right)^{-1}$, then $\mu_{n}(g)=U_{n}(z), \nu_{n}(g)=L_{n}\left(z ; e^{i t}\right)+$ $n^{2} U_{n}(z) c_{n}$, and $L_{n}(z ; g)=K_{n}^{(2)}(z)$. Writing $K_{n}^{(3)}(z)=K_{n}^{(3)}(z ; g)$, the equation (4.6) gives

$$
\begin{aligned}
-K_{n}^{(3)}(z)-n\left(2 U_{n}(z)-r(z)\right) K_{n}^{(2)}(z)+ & \frac{\gamma_{n} n}{2} L_{n}\left(z ; e^{i t}\right) \\
& -n z U_{n}^{\prime}(z)-\frac{n z^{2}}{2} U_{n}^{\prime \prime}(z)=0,
\end{aligned}
$$

after some simplification. Next, we choose $g(t)=e^{i t}$ in (4.6). Then $\mu_{n}(g)=c_{n}$ and $\nu_{n}(g)$ is independent of $z$. Equation (4.6) becomes

$$
-K_{n}^{(3)}\left(z ; e^{i t}\right)-n\left(2 U_{n}(z)-r(z)\right) L_{n}\left(z ; e^{i t}\right)+f_{n}+n z U_{n}(z)=0
$$

where $f_{n}=-\gamma_{n} n^{3} c_{n}^{2} / 2+\gamma_{n} n \nu_{n}\left(e^{i t}\right) / 2+n c_{n}$.

Multiply equation (4.7) with $2 U_{n}(z)-r(z)$ and use equation (4.8) to get

$$
\begin{aligned}
-\left(2 U_{n}(z)-r(z)\right) K_{n}^{(3)}(z)+\frac{\gamma_{n}}{2}\left[-K_{n}^{(3)}\left(z ; e^{i t}\right)+f_{n}+n U_{n}(z)\right] \\
-n\left(2 U_{n}(z)-r(z)\right)^{2} K_{n}^{(2)}(z)-n\left(z U_{n}^{\prime}(z)+\frac{z^{2}}{2} U_{n}^{\prime \prime}(z)\right)\left(2_{U} n(z)-r(z)\right)=0 .
\end{aligned}
$$

Note that

$$
\frac{\gamma_{n} z}{2} U^{\gamma_{n}}(z)-\left[z\left(U^{\gamma_{n}}\right)^{\prime}(z)+\frac{z^{2}}{2}\left(U^{\gamma_{n}}\right)^{\prime \prime}(z)\right]\left(2 U^{\gamma_{n}}(z)-r(z)\right)=0
$$

Multiply equation (4.10) with $n$ and subtract from equation (4.9). This gives the following equation

$$
\begin{aligned}
-\frac{2}{n} D_{n}(z) K_{n}^{(3)}(z)-s(z) & K_{n}^{(3)}(z)-n\left(\frac{2}{n} D_{n}(z)+s(z)\right)^{2} K_{n}^{(2)}(z) \\
+\frac{\gamma_{n}}{2} K_{n}^{(3)}\left(z ; e^{i t}\right) & +\frac{\gamma_{n}}{2}\left(f_{n}+D_{n}(z)+z D_{n}(z)\right) \\
+ & \left(\frac{2}{n} D_{n}(z)+s(z)\right)\left(z D_{n}^{\prime}(z)+\frac{z^{2}}{2} D_{n}^{\prime \prime}(z)\right)=0
\end{aligned}
$$

where $s(z)=\gamma_{n}(z+1 / z) / 2+1$. This equation is the basis for the 
Proof of lemma 3.4. We use the same notation as in the proof of lemma 3.3. Note that

$$
K_{n}^{(3)}(z)=\frac{\partial^{3} F_{n}}{\partial w_{2}^{3}}-3 \frac{\partial^{2} F_{n}}{\partial w_{2}^{2}} \frac{\partial F_{n}}{\partial w_{2}}+2\left(\frac{\partial F_{n}}{\partial w_{2}}\right)^{3},
$$

and

$$
K_{n}^{(3)}\left(z ; e^{i t}\right)=\frac{\partial^{3} F_{n}}{\partial w_{2}^{2} \partial w_{1}}-\frac{\partial^{2} F_{n}}{\partial w_{2}^{2}} \frac{\partial F_{n}}{\partial w_{1}}-2 \frac{\partial^{2} F_{n}}{\partial w_{1} \partial w_{2}} \frac{\partial F_{n}}{\partial w_{2}}+2\left(\frac{\partial F_{n}}{\partial w_{2}}\right)^{2} \frac{\partial F_{n}}{\partial w_{1}},
$$

where all derivatives are evaluated at the point $(z, 0,0)$. Cauchy's integral formula and inequality (4.4) now yield

$$
\left|K_{n}^{(3)}(z)\right| \leq C \quad \text { and } \quad\left|K_{n}^{(3)}\left(z ; e^{i t}\right)\right| \leq C .
$$

If we use (3.20), Cauchy's integral formula to estimate $D_{n}^{\prime}$ and $D_{n}^{\prime \prime}$, the estimates (4.12) and take $z=z_{n}$ in (4.11) $\left(s\left(z_{n}\right)=0\right)$, we get

$$
\left|\frac{\gamma_{n} f_{n}}{2}\right| \leq C
$$

if $n \geq n_{0}$. Now, use equation (4.11) again with $|z|=1-3 \delta / 2$ together with (3.20) and (4.11) - (4.13) to see that $\left|n K_{n}^{(2)}(z)\right| \leq C$ if $n \geq n_{0}$ and $|z|=1-3 \delta / 2$. Since $K_{n}^{\gamma_{n}, 0}(z)=-K_{n}^{(2)}(z)$ lemma 3.4 follows using the maximum principle.

The rest of this section is devoted to the proofs of the lemmas 2.4, 2.5 and 3.2 .

Proof of lemma 2.4. Denote a permutation in $S_{N}$ by $\pi^{(N)}$ and put $g_{n}\left(\pi^{(N)}\right)=1$ if $\pi^{(N)}$ does not have an increasing subsequence of length $>n$ and $g_{n}\left(\pi^{(N)}\right)=0$ otherwise. Clearly,

$$
F_{N}(n)=\frac{1}{N !} \sum_{\pi^{(N)} \in S_{N}} g_{n}\left(\pi^{(N)}\right) .
$$

Let $S_{N+1}(k)$ denote the set of all $\pi^{(N+1)}$ such that $\pi^{(N+1)}(1)=k, k=1, \ldots, N+$ 1. Each $S_{N+1}(k)$ contains $N$ ! elements and we can define a bijection $F_{k}$ : $S_{N+1}(k) \rightarrow S_{N}$ as follows. Let $\psi_{k}(m)=m$ if $1 \leq m \leq k-1$ and $\psi_{k}(m)=m-1$ if $k+1 \leq m \leq N+1$, so that $\psi_{k}$ is an increasing map from $\{1,2, \ldots, k-1, k+$ $1, \ldots, N+1\}$ to $\{1, \ldots, N\}$. Put

$$
F_{k}\left(\pi^{(N+1)}\right)(m)=\psi_{k}\left(\pi^{(N+1)}(m+1)\right), \quad m=1, \ldots, N,
$$

for $\pi^{(N+1)} \in S_{N+1}(k)$. Clearly, $F_{k}\left(\pi^{(N+1)}\right)$ maps $\{1, \ldots, N\}$ into itself and, since $\psi_{k}$ is strictly increasing, $F_{k}\left(\pi^{(N+1)}\right) \in S_{N}$. Also, $F_{k}: S_{N+1}(k) \rightarrow S_{N}$ is a bijection. Note that if $F_{k}\left(\pi^{(N+1)}\right)$ has an increasing subsequence of length 
$>n$, then $\pi^{(N+1)}$ has an increasing subsequence of length $>n$, because adding $\pi^{(N+1)}(1)=k$ in the beginning can only increase the length of the increasing subsequence; recall that $\psi_{k}$ is strictly increasing. Hence

$$
g_{n}\left(F_{k}\left(\pi^{(N+1)}\right)\right) \geq g_{n}\left(\pi^{(N+1)}\right) .
$$

From (4.15) we get

$$
\begin{aligned}
\frac{1}{(N+1) !} \sum_{\pi^{(N+1)} \in S_{N+1}} g_{n}\left(\pi^{(N+1)}\right) & =\frac{1}{(N+1) !} \sum_{k=1}^{N+1} \sum_{\pi^{(N+1)} \in S_{N+1}(k)} g_{n}\left(\pi^{(N+1)}\right) \\
& \leq \frac{1}{(N+1) !} \sum_{k=1}^{N+1} \sum_{\pi^{(N+1)} \in S_{N+1}(k)} g_{n}\left(F_{k}\left(\pi^{(N+1)}\right)\right) \\
& =\frac{1}{(N+1) !} \sum_{k=1}^{N+1} \sum_{\pi^{(N)} \in S_{N}} g_{n}\left(\pi^{(N)}\right),
\end{aligned}
$$

and hence using (4.14) the lemma is proved.

Proof of lemma 2.5. Write $w_{N}(\lambda)=\lambda^{N} e^{-\lambda} / N$ !. By Stirling's formula we have

$$
w_{N}(\lambda) \sim \exp \left(-\lambda\left(\frac{N}{\lambda} \log \frac{N}{\lambda}+1-\frac{N}{\lambda}\right)\right) \frac{1}{\sqrt{2 \pi N}} .
$$

Put $f(x)=x \log x+1-x$. Then

$$
w_{N}(\lambda) \leq C \exp (-\lambda f(N / \lambda)) .
$$

If $0 \leq x \leq 2$ it is easy to show that $f(x) \geq(x-1)^{2} / 4$, and consequently

$$
w_{N}(\lambda) \leq C \exp \left(-\frac{\lambda}{4}\left(\frac{N}{\lambda}-1\right)^{2}\right)
$$

if $0 \leq N \leq 2 \lambda$. Also, $f(x) \geq x / 10$ if $x \geq 2$ and hence

$$
w_{N}(\lambda) \leq C e^{-N / 10},
$$

if $N \geq 2 \lambda$. The inequality (4.17) gives

$$
\sum_{N \geq 2 \lambda} w_{N}(\lambda) \leq C e^{-\lambda / 5}
$$

From (4.16) we get

$$
\sum_{N \leq \lambda-3 \sqrt{\lambda \log \lambda}} w_{N}(\lambda) \leq \frac{C}{\lambda^{2}},
$$


and

$$
\sum_{\lambda+3 \sqrt{\lambda \log \lambda} \leq N \leq 2 \lambda} w_{N}(\lambda) \leq \frac{C}{\lambda^{2}} .
$$

Since $0 \leq F_{N}(n) \leq 1$, we obtain

$$
\left|\sum_{|N-\lambda| \leq 3 \sqrt{\lambda \log \lambda}} w_{N}(\lambda) F_{N}(n)-\phi_{N}(\lambda)\right| \leq \frac{C}{\lambda^{2}},
$$

for $\lambda$ sufficiently large and $0 \leq n \leq N$. Now, since $F_{N}(n)$ is decreasing in $N$,

$$
\sum_{|N-\lambda| \leq 3 \sqrt{\lambda \log \lambda}} w_{N}(\lambda) F_{N}(n) \leq F_{\nu(\lambda)}(n)
$$

where $\nu(\lambda)=[\lambda-3 \sqrt{\lambda \log \lambda}]$, and

$$
\sum_{|N-\lambda| \leq 3 \sqrt{\lambda \log \lambda}} w_{N}(\lambda) F_{N}(n) \geq F_{\mu(\lambda)}(n)-\frac{C}{\lambda^{2}},
$$

where $\mu(\lambda)=[\lambda+3 \sqrt{\lambda \log \lambda}]$. If we choose $\lambda=\mu_{N}$, then $\nu\left(\mu_{N}\right) \geq N$ if $N$ is sufficiently large, and hence combining (4.18), (4.19) and lemma 2.4 we get the left inequality in (2.6). If we take $\lambda=\nu_{N}$, then $\mu\left(\nu_{N}\right) \leq N$, if $N$ is sufficiently large. Combining (4.18), (4.20) and lemma 2.4 we get the right inequality in (2.6). The lemma is proved.

Proof of lemma 3.2. The function $U(z)$ is analytic in $\mathbb{C} \backslash \mathbb{T}, \mathbb{T}$ the unit circle and solving equation (3.6) we get

$$
U(z)=\frac{1}{2}\left[\frac{\xi}{2}\left(z-\frac{1}{z}\right)+1+\sqrt{\left(\frac{\xi}{2}\left(z+\frac{1}{z}\right)+1\right)^{2}+2 \xi c-\xi^{2}}\right]
$$

and

$$
U(z)=\int_{-\pi}^{\pi} \frac{u(t)}{1-z e^{-i t}} d t
$$

where $u(t)$ is a probability density given by

$$
u(t)=\lim _{r \rightarrow 1-} \frac{1}{2 \pi} \operatorname{Re}\left(2 U\left(r e^{i t}\right)-1\right) .
$$

From (4.21) we get

$$
2 U(z)-1=\frac{\xi}{2}\left(z-\frac{1}{z}\right)+\sqrt{\left(\frac{\xi}{2}\left(z+\frac{1}{z}\right)+1\right)^{2}-b^{2}},
$$


where we have written $2 \xi c-\xi^{2}=-b^{2}$. If we define $w=\xi(z+1 / z) / 2+1$, then $\sqrt{w^{2}-b^{2}}$ must be defined so that $2 U(z)-1$ is analytic in $\mathbb{C} \backslash \mathbb{T}$. The image of $\mathbb{T}$ under $z \rightarrow w,[1-\xi, 1+\xi]$ must cover the cut $[-b, b]$ in the $w$-plane, so $b$ is real $\geq 0$ and

$$
[-b, b] \subseteq[1-\xi, 1+\xi]
$$

unless $b=0$. If $\xi \leq 1$, then $b=0$ and $2 U(z)-1=1+\xi z$, which gives $u(t)=(1+\xi \cos t) / 2 \pi$ and $c=\xi / 2$. If $\xi>1$ the inlusion (4.22) gives the inequality $b \leq \xi-1$. Assume that $b<\xi-1$. Write $\sqrt{w^{2}-b^{2}}=\sqrt{w+b} \sqrt{w-b}$. $\mathrm{Az} z \rightarrow 0$ along the real axis, $w \rightarrow+\infty$ along the real axis, and since $2 U(z)-1$ is bounded and $\xi(z-1 / z) / 2 \rightarrow-\infty$, both roots must be real and $\geq 0$ when $w>b$. Thus we can take the arguments of $w-b$ and $w+b$ between $-\pi$ and $\pi$. If $z=r e^{i \theta}$ and $0 \leq \theta \leq \pi$, then $w$ approaches $[1-\xi, 1+\xi]$ from the lower half-plane as $r \rightarrow 1-$. Since $b<\xi-1$ we can choose $\theta_{0}$ in $[0, \pi]$ so that $1-\xi<1+\xi \cos \theta_{0}<-b$, and we get

$$
\sqrt{w^{2}-b^{2}} \rightarrow\left|\left(1+\xi \cos \theta_{0}\right)^{2}-b^{2}\right|(-i)^{2}
$$

as $r \rightarrow 1-$, which gives $u\left(\theta_{0}\right)<0$ This contradiction shows that $b=\xi-1$ if $\xi>1$. Thus $2 \xi c-\xi^{2}=-(\xi-1)^{2}$, which gives $c=1-1 / 2 \xi$ and a straightforward computation gives $u(\theta)$.

\section{Acknowledgement}

I thank Persi Diaconis for drawing my attention to problems on random permutations and for warm hospitality during a visit to Harvard.

\section{References}

[AD] D. Aldous and P. Diaconis, Hammersley's interacting particle process and longest increasing subsequences, Prob. Th. and Rel. Fields 103 (1995), 199-213.

[BB] R. M. Baer and P. Brock, Natural sorting over permutation spaces, Math. Comp. 22 (1968), 385-410.

[BDJ] J. Baik, P. A. Deift, and K. Johansson, On the variance of the longest increasing subsequence in a random permutation, In preparation.

[DZ1] J.-D. Deuschel and O. Zeitouni, Limiting curves fot i. i. d. records, Ann. Probab. 23 (1995), 852 - 878.

[DZ2] - On increasing subsequences of i.i.d. samples, preprint.

[Ge] I. M. Gessel, Symmetric functions and P-recursiveness, J. Combin. Theory Ser. A 53 (1990), 257-285.

[GWW] I. Gessel, J. Weinstein, and H. S. Wilf, Lattice walks in $\mathbb{Z}^{d}$ and permutations with no long ascending subsequences, Electr. J. Comb. 5 (1998).

[GW] D. J. Gross and E. Witten, Possible third-order phase transition in the large- $N$ lattice gauge theory, Phys. Rew. D, 21 (1980), 446-453.

[Ha] J. M. Hammersley, A few seedlings of research, Proc. Sixth Berkeley Symp. Math. Statist. and Probability, vol. 1, University of California Press, 1972, pp. 345-394. 
[Jo] K. Johansson, On fluctuations of eigenvalues of random Hermitian matrices, Duke Math. J. (1998).

[Ki] J.-H. Kim, On the longest increasing subsequence of random permutations - a concentration result, J. Comb. Th. A 76 (1996), 148-155.

[LS] B. F. Logan and L. A. Shepp, A variational problem for random Young tableaux, Adv. Math. 26 (1977), 206-222.

[Me] M. L. Mehta, Random matrices, 2nd ed., Academic Press, San Diego, 1991.

[Od] A. M. Odlyzko, personal communication.

[OPWW] A. M. Odlyzko, B. Poonen, H. Widom, and H. Wilf, On the distribution of longest increasing subsequences in random permutations, in preparation.

[OR] A. M. Odlyzko and E. Rains, On longest increasing subsequences in random permutations, in preparation.

[PS] V. Periwal and D. Shevitz, Unitary-matrix models as exactly solvable string theories, Phys. Rev. Lett. 64 (1990), 1326-1329.

[Ra] E. M. Rains, Increasing subsequences and the classical groups, Electron. J. of Combinatorics 5(1) (1998), R12.

[Se1] T. Seppäläinen, A microscopic model for the Burgers equation and longest increasing subsequences, Electron. J. Prob. 1 (1996).

[Se2] L Large deviations for increasing subsequences on the plane, preprint (1997).

[Sz1] G. Szegö, Orthogonal polynomials, vol. XXII, Amer. Math. Soc. Colloquium Publ., New York, 1939.

[Sz2] On Certain Hermitian forms associated with the Fourier series of a positive function, Comm. Seminaire Math. de l'Univ. de Lund, tome supplémentaire, dédié a Marcel Riesz (1952), 228-237.

[U] S. M. Ulam, Monte Carlo calculations in problems of mathematical physics, Modern mathematics for the engineer (E. F. Beckenbach, ed.), McGraw-Hill, 1961, pp. 261281.

[VK] A. M. Vershik and S. V. Kerov, Asymptotics of the Plancherel measure of the symmetric group and the limiting form of Young tables, Soviet Math. Dokl. 18 (1977), 527-531.

[We] H. Weyl, The classical groups, Princeton University Press, Princeton, 1946.

Dept. of Math., Royal Institute of Technology, S-100 44 Stockholm, SWEDEN

E-mail address: kurtj@math.kth.se 